\title{
La Clasificación de Atlas y Mapas
}

Roberto Abell B.

Investigador del CUIB

\section{RESUMEN}

Se ha cam bia do los es que mas y las ta blas que las acom pa ñan para atlas y ma pas en la Bi blioteca del Con gresoy por eso re quieren un nue vo análi sis, to ma do de un Manual para la aplica ción de la Cla sifica ción del Con gre soa lageo grafía, an tro polo gíay recreación, clase $G$ publica dore cien te men te en es pañol. La tabla III para re gio nes y países con bloques de nú me ros es la ta bla base para el de sa rro llo de los nú me ros de cla sificación pero la tabla IV para atlas y ma pas de ma te ria se ela bo ra en for ma es pe cial y se asig na una le tra y nú mero(s) que pa re cen un ter cer Cutter pero no lo es.

Exis ten otros es que mas que son ca sos es pe cia les en Mé xi co con la ex cep ción de la Cla sificación de Dewey (DD) que se utiliza en las bibliotecas públicas con colecciones no especialmente grandes y complejas.

\section{ABSTRAC}

The sche du les and the ta bles that accomp nay them for atlas and maps in the Li brary of Con gress have been changed and therefore require a fresh analysis, taken from a Manual for the application of the Library of Congress Classification for geography, anthropology and recreation, class $G$ published recently in Spanish. Tabla III for re gions and coun tries with blocks of num bers is the ba sic ta ble for the de ve lopment of classification numbersbut table IV for subject atlas and maps is elaborated in a special manner and is assigned a letter and number(s) that appear to be a third Cutter but is not.

Other sche du les exist which are spe cial ca ses in Me xi co with the ex cep tion of the Dewey DecimalClassification (DD) which is used in public libraries with collections not especially large and complex.

\section{INTRODUCCION}

Mu chas bi blio te cas ad quie ren atlas y ma pas y por los da tos geográficos va lio sos que con tie nen es nece sa rio ha cer los lle gar a las ma nos de los usua rios. En for ma vi sual los atlas y ma pas abar can información que un texto no puede comunicar adecuadamente. Tienen uso no sólo para geógrafos en sus investigaciones sino, por ejemplo, ayudan a la planeación industrial para ubicarse en de ter minadolugar. Divulgan de talles que pue den pre ci sardón de se establece más convenientemente una zona industrial de las dependencias gubernamentales o de la iniciativa privada.

La Clasificación del Congreso es, tal vez, la más conocida y la más com ple ta. Los nú me ros de cla si fi ca ción loca li zan a los atlas y ma pas en un sólo lu gar, en tre la sub cla se G1000- 9980 (Li brary of Con gress 1976, p. 12-223). Ade más, cabe en tre la his to ria por un lado y la an tro po lo gía como cien cia so cial por otro, y sir ve de puente entre las dos y las que la siguen. Especialmente para los mapas con su formato propio, los números juntos resultan conve nien tes para se pa rar los y co lo car los en un solo si tio. Como requieren atención cuidadosa que obliga su montaje y su colocación en perchas o muebles especiales, con referencias en el catálogopúblico, pueden dirigir al usuario a una sección audiovi sual en don de se en cuen tra un es pa cio ade cua do para su uso.

El es que ma y las ta blas para la cla si fi ca ción de atlas y ma pas han sido cam bia das en tre la ter ce ra y cuar ta edi ción de la cla se G (Library of Con gress 1954, p. 14- 183; Li brary of Con gress 1976, p. 12-223) y por lo tan to re quie ren un nue vo aná li sis so bre las mismas. Como todo se renueva dentro de las ciencias, esto es motivo para estas innovacionesrespecto al campo de la geografíade atlas y mapas. Por ejemplo, existen nuevos números para la exploración y estudio del universo exterior. También las nuevas subdivisiones geo gráficas refle jan los cambios políticos des de la 
Se gun da Gue rra Mun dial, es pe cial men te re fe ren te a Asiay Afri ca. Las ta blas in clu yen mu chos te mas nue vos no de talla dos an teriormente. Además, en las adiciones y cambios constantemente apa re cen nue vos asun tos para el de sa rro llo de la cla sifica ción de atlas y mapas (Library of Congress 1928- ).

Las tablas para estos materiales visuales presentan dificultades al cla sifica dor que tie ne que or de nar los co rrec ta men te o al maestro que ne ce si ta en se ñar a sus alum nos cómo uti li zar los. Tal vez sean entre las más difíciles y detalladas de la Clasificación del Congreso que requieren un análisis más detallado. La guía de Immroth (1980, p. 101-102,172-179) tie ne una orien ta ción re feren te al mun do an gloame ri ca no y el tra ba jo de Lars gaard (1978) es casi semejante, además de tener otro enfoque. El estudio del autor (Abell 1977, p. 122-150) resulta ya obsoleto por estar basado en la tercera edición del esquema de la clase G (Library of Congress 1954).

En términos generales, se procedió al esbozo de la estructura y las carac te rís ticas es pe cia les deles que ma se gún elestu dio de las mismas y de las fuentes documentales existentes. Después se bus ca ron las fi chas bi bliográficas parailus trar en for ma prác tica el trabajo. Lue go se in ves ti gó so bre las nor mas ca ta lo grá ficas de $R C A-2$ (1983), se obtuvieron los puntos de acceso necesarios para la recuperación completa de la información. Esto incluye los temas que se recogen según el Instituto Colombiano de Fomen to de la Edu ca ción Su pe rior (IN FES) (1985) y como adi ción se aprovechó el de Escamilla (1978) y Bilindex (1984).

\section{LA CLASIFICACION}

La clasificaciónde los atlas y ma pas se en cuen tra den tro del esquema de geografía con que empieza la clase G. Después de la geo gra fía en ge ne ral que se ini cia con di vi siones de for ma, viajes $\mathrm{y}$ tu ris mo, his to ria de los des cu bri mien tos y ex plo ra cio nes mundia les, geo gra fía ár ti ca, an tár ti ca, de los tró pi cos y de los he misfe rios nor te y sur, em pie za la sec ción so bre atlas, glo bos y ma pas propiamente dicha.

Se utilizan tablas auxiliares combinadas para clasificar estos materiales geográficamente, auxiliares por encontrarse al final de la sub cla se $\mathrm{G}$ y no den tro de este es que ma y com bi na das por contenerelementosgenerales, te máticos y sobrelocalidades.El esquema desglosa bloques de tres, cuatro y cinco números por regiones, países y estados de todo el mundo en un largo listado ocupando casi doscientas hojas, para atlas, globos y mapas, antes de las cuatro tablas mismas. Además se encuentra con este des glo se de nú me ros y blo ques de nú me ros acom pa ña dos, en su caso, de cartasilustrativas de diferentes regiones del mun do entre el esquema como guía para el clasificador. Para la historia y descripción de atlas y mapas, se refiere al clasificador a la subclase GA sobre cartografía.

Para cada bloque de números geográficos de los atlas, globos y mapas en sí, se utilizan unas tablas bastante detalladas y extensas, al fi nal de los es que mas de la sub cla se $\mathrm{G}$ (véan se cua dros 1 y 2). La tabla I da ejemplos exclusivamente sobre la construcción de la signatura to po grá fi ca del ma te rial, y la ta bla II el or den topográfico de las fichas catalográficas. La tabla III, acompañada con instrucciones y ejemplos, desglosa la asignación propiamen te di cha de cada nú me ro de los blo ques den tro del es que ma. La ta bla IV tam bién es bá si ca, con su lis ta do de sub di vi sio nes de te mas en caso de que se cla si fi que el atlas o el mapa no sólo por área geográfica sino también por materia.

Encontrado el bloque de números del área geográfica o cuerpo ce les te del atlas, glo bo o mapa, se pro ce de a se lec cio nar el nú mero es pe cífico de cla si fica ción se gún la ta bla III. Si elúl ti mo dígi to del pri mer nú me ro del blo que ter mi na en cero (" 0 "), se uti li za la pri me ra co lum na de nú me ros de la ta bla III, 0 a 4 , sumando la ci fra es co gi da al nú me ro base del blo que. Al con tra rio, si la úl tima ci fra del pri mer nú me ro del blo que ter mi na en " 5 ", se di ri ge a la se gun da co lum na de nú me ros de la mis ma ta bla, 5 a 9, sustituyendo la cifra apropiada de la tabla por el número correspondien te del blo que geo grá fi co. Se re cu rre tam bién a la ta bla IV de ma te rias, siem pre y cuan do el atlas o mapa tra te del área com pleta en cuestión, sumando el número " 1 ", en su caso, al número base del bloque o sustituyendo el número " 6 " por el número equivalente del bloque. También para los siguientes tres números de la tabla III so bre re giones, di vi siones po líticas mayores y lugares respectivamente se utiliza, al mis mo tiem po, la tabla IV cuando el ma te rial se li mi ta a un tema es pe cífico, pero nun ca se apli ca la ta bla IV a los atlas, glo bos o car tas ge ne ra les. En se guida se verá como se clasifican estos materiales según las dos tablas en cuestión. No se han incluido ejemplos sobre globos por ser su tratamiento igual a ma pas y atlas, ade más de ser poco frecuente su procesamiento en las bibliotecas. 


$$
\begin{aligned}
& 1540-1542 \\
& 1565-1549 \\
& 1550-1552 \\
& 1600-1602 \\
& 1605-1609 \\
& 1700-1702 \\
& 1775-1779
\end{aligned}
$$

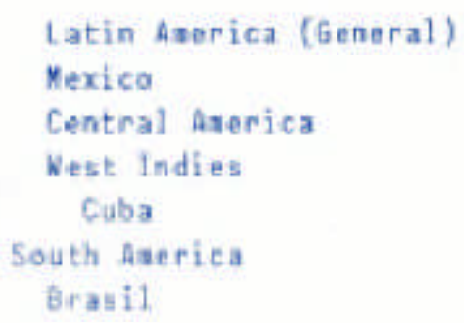

\section{MAPS}

5y region or country

Aserica. Western Heaisphers

North Aeerica United States

Mexico

Litier and touns, $A-Z$

e. g. .ME Mexico City

$4420=4+422$

$4510-4513$

$4520-4523$

$4530-4532$

$4580-4583$

$4680-4682$

4682

$4690-4693$

$5200-5202$

$5280-5286$

$5290-5296$
Nopthern States

Zacatecas

San Luis Potost

Central States Mexico (State)

Southern States

Regiens, natural features, etc., A-Z e. g. . Th Isthaus of Tehuantepec Euerrero

South Anerica (Kap, p. 40)

Venezuela

Colombia

Guadro $?$

SPECIAL INSTRUCIIOYS

III

\section{AREA SUBDTVISIONS}

Each sequence of two or nore nunbers assigned to a geographic area is subdivided in accordance

with the following plan:

(1) 0 or 5 General

(2) 1 er 6 By subjegst

Subarranged by Table IV

(3) 2 or 7 By region, natural feature, ete., when, not assigned individual nuabers, $A-2^{2}$

(4) 3 or 8 . Oy najor political division (Counties, staten, provinces, etc.) when not assigned individal nutubers, $h-2$

(5) 4 op 9 By city or toun, ta-z -Al Cities (Collective)

c. g. 01819.A1. At las of cities of England

Cities and touns of aost countries are grouped under the country, not under the political subdivision in which they are located. 


\section{IV}

\section{SUBJECI SUBDIVISIONS}

Summary of Form and Subject Subdivisions

A Special category nape and atlases

B Mathematical geography

C Physical sciences

D Biogeography

E Hunan and cultural geography. Anthropogeography, Human ecology

$F$ Political geography

$G$ Economic geography

$H$ Mines and mineral resources

$J$ Agriculture

$K$ Forests and forestry

$L$ Aquatic biological resources

$M$ Manufacturing and processing. Service industries

N Technology, Engineering, Public works

P Iransportation and communication

$Q$ Conmerce and trade. Finance

R Military and naval geography

S Historical geography

Physical Sciences

Class here works on the distribution of natural phenomens of the earth, the atmosphere, and subsurface features

.C5 Geology

.C51 Geological surveys. Status. Progress

.C55 Dynanic and structural geology. Tectonics. Earthquakes (Seismology). Vulcanology

.C8 Meteorology and climatology. Climate classification systems

Human and Cultural Geography. Anthropogeography. Huinan Ecology

Class here works that are concerned with man

.E1 General as a physical and social being

Including ethnology, tribes, ethnic groups, etc.

.E15 Archaeological sites. Cities and towns which are ruined, extinct, etc.

.E2 Population

P

Transportation and Communication

.P3 Railroads

.P55 Ports and port facilities

.P6 Air transportation. Aeronautical charts 
Cuadro 3

TABLAS DE NUMEROS DE CUTTER

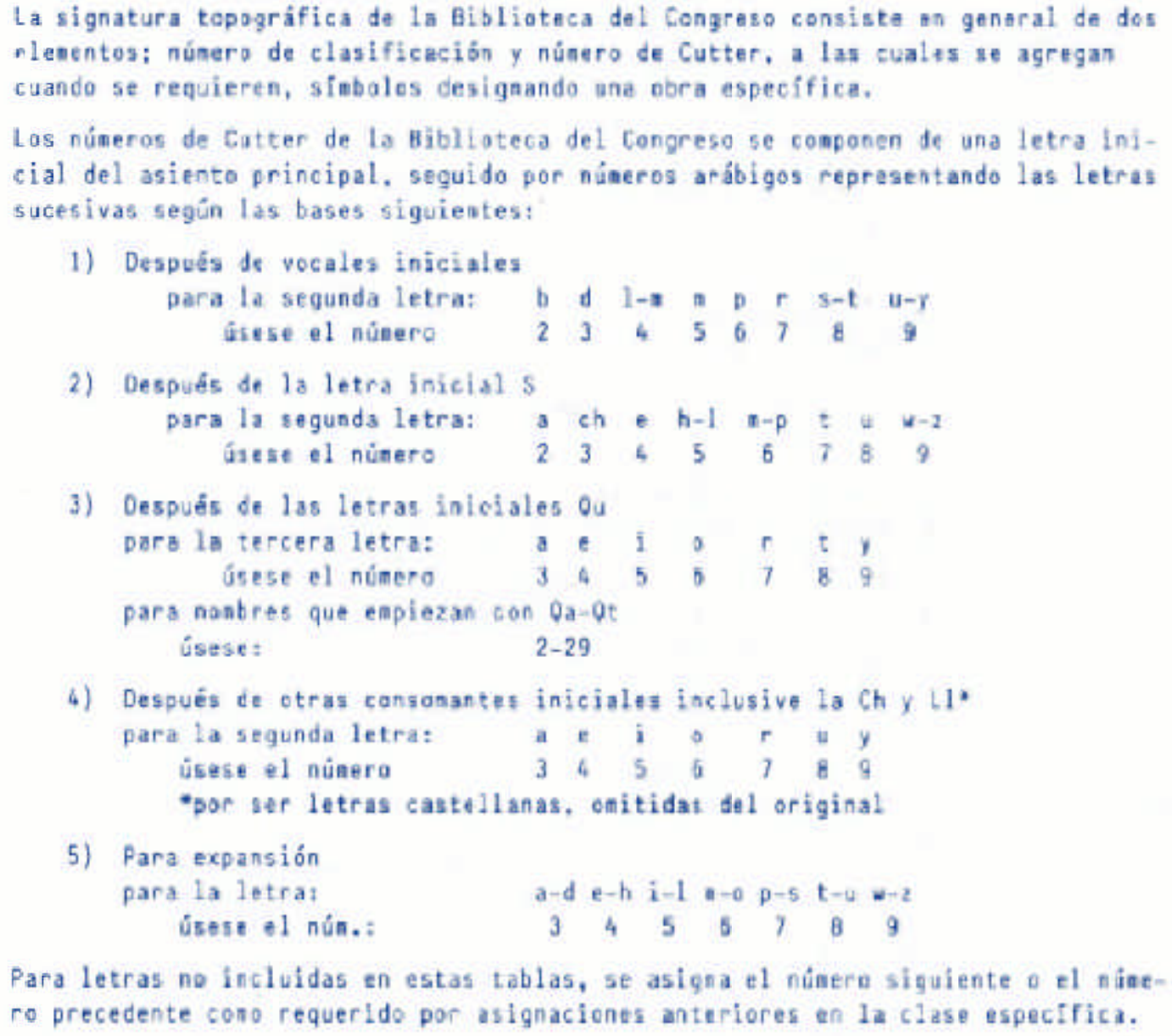

Para letras no incluidas en estas tablas, se asigna el número siguiente o el ninero precedente cono requerldo por asignaciones anteriores en la clase espectfica.

El primer número de la tabla III (0 ó 5) se escoge para mapas y atlas generales y topográficos del área en cuestión. Lasignatura topográfica consiste de tres elementos:
Para atlas

- Clasificación por área

- Número de Cutter de la autoridad responsable

- Fecha de publicación

\section{Para mapas}

- Clasificación por área

- Fecha

- Número de Cutter de la autoridad responsable
La fe cha es co gi da para los ma pas no es ne ce sa ria men te la fe cha de pu bli ca ción sino la fe cha que re pre sen tan los da tos del mapa, aun que mu chas ve ces coin ci den, a me nos que sea un mapa his tóri co. En tal caso se asig na la fe cha de pu bli ca ción. La au to ri dad no es for zo sa men te el asien to prin ci pal sino el res pon sa ble de la compilación o publicación del mapa. A continuación se ilustra esta categoría de atlas y mapas generales con los siguientes

ejemplos y ade más con la ex pli ca ción de los di fe ren tes ele mentos de que está compuesta la signatura topográfica:

\section{Ejemplo 16}

G1545

.G36

1977

G1545
García de Miranda, En riqueta Atlas, nuevo atlas Porrúa de la Re pú blica Mexicana / Enriqueta García de Miranda, Zaida Fal cón de Gyves. - 3a. ed. - Es ca las difieren. - México : Porrúa, 1977 latín (197 p.) : mapas col. ; $35 \mathrm{~cm}$.

1. México - Mapas. I. Falcón de Gyves, Zaida. II. Título. III. Título: Nuevo atlas Porrúa de la República Mexicana

El nú me ro de cla si fi ca ción para atlas de Mé xi co, escogiendo el " 5 " de la segunda columna de números de la tabla III por ser un atlas general 
.G36 El número de Cutter del asiento según la tabla 4 de au to res para el pri mer nú me ro y la ta bla 5 para el segun do ajus ta do para pre ser varel or den alfabéti co de asientos en el catálogo topográfico (Cuadro 3)

1977 Fecha de publicación

\section{Ejemplo 17}

G1580

1962

.T3

G4580

El nú me ro de cla si fica ción para ma pas delEs ta do de México, es co gien do el " 0 " de la pri me ra colum nade números de la tabla III por ser mapa general

1962 Fecha por los datos representados

.T3 El nú me ro de Cut ter del res pon sa ble del mapa se gún la tabla 4 de autores

\section{Ejemplo 18}

G5290

1958

. $\mathrm{I} 5$

$$
\begin{aligned}
& \text { Instituto Geográfico Agustín Codazzi Re- } \\
& \text { públicadeColombia:mapafísicopolí- } \\
& \text { tico / elaborado por el Instituto } \\
& \text { Geográfico de Colombia Agustín Co- } \\
& \text { dazzi. - Escala } 1: 1,500,000 \\
& \text { (N } \left.430 \text { '56" } 57 / 07404^{\prime} 51 ", 3\right) .- \text { [Bogo- } \\
& \text { tá] : El Instituto, } 1958 \\
& 1 \text { mapa en pliegos : col. ; } 75 \times 119 \\
& \text { cm. cada pliego }
\end{aligned}
$$

1. Colombia - mapas. I. Título

G5290 Elnúmerodeclasifica ción para mapas de Colombia, escogiendo el " 0 " de la primera columna de la tabla III por ser un mapa general

$1958 \quad$ Fecha por los datos representados

.15

El número de Cutter según la tabla I de autores

Se se lec cio na el se gun do nú me ro de la ta bla III (1 ó 6) para atlas y ma pas cuan do se tra te de al gu na materia específica, siendoun enfoquelinguístico,económico,tecnológico, histórico,etcétera. Por lo tanto, se complementa la clasificación escogiendo de la tabla IV la letra jun to con el nú me ro se gún el tema. Esta com bi- nación de letra y número(s) no es un número de Cutter. La signatura topográfica para un atlas de materia consiste de tres elementos y para un mapa, de cuatro divisiones:
Para atlas

- Clasificación por área

- Letra y número de materia + número de Cutter de la autoridad responsable

- Fecha de publicación

\section{Para mapas}

- Letra y número de materia

- Fecha

- Número de Cutter de la autoridad responsable
- Clasificación por área

\section{Ejemplo 19}

G1606

.E2

C8

1979

G1606

. $\mathrm{E} 2$

C8

1979

\section{Ejemplo 20}

G1414

.C5

1961

. 77

\section{G4411}

. $\mathrm{C} 5$
Cuba. Co mi té Es ta tal de Es ta dís ticas Atlas demográfico de Cuba / República de Cuba, Comité Estatal de Estadísticas. - Escala 1:2,750,000. - [La Habana] : República de cuba, Instituto Cubano de Geodesia y Cartografía, 1979

1 atlas (99 p.) : il. col., mapas col. ; $33 \mathrm{~cm}$.

1. Cuba - Po bla ción - Ma pas. I. InstitutoCubano de Geodesia y Cartografía. II. Título.

El número de clasificación para atlas de Cuba, de materia, sustituido del segundo número del bloque, el "6" de la se gun da co lum na de la ta bla III por ser su último dígito del primer número del bloque el "5"

La letra y el número de la tabla IV para materiales cartográficos sobre población

El número de Cutter del asiento según la tabla 4 de autores (cuadro)

Fecha de publicación
Por terminar el primer número del bloque para mapas de México en " 0 ", se escoge de la primera columna de la tabla III el número "1" para mapas de materia y se le suma al número base del bloque

La letra y el número escogidos de la tabla IV para materiales cartográficos sobre geología 


\begin{tabular}{|c|c|}
\hline 1961 & Fecha \\
\hline. $\mathrm{C} 7$ & $\begin{array}{l}\text { El nú me ro de Cut ter del asien to se gún la ta bla IV de } \\
\text { autores }\end{array}$ \\
\hline \multicolumn{2}{|c|}{ Ejemplo 21} \\
\hline $\begin{array}{l}\text { G4421 } \\
. \mathrm{P} 3 \\
1958 \\
. \mathrm{M} 4\end{array}$ & $\begin{array}{l}\text { México. Dirección Ge neraldeConstruccio- } \\
\text { nes de Ferrocarriles. Ferrocarril Chi- } \\
\text { huahua-Pacífico / S.C.O.P., Dir. Gral. } \\
\text { de Const. de FF.CC. - Escala } \\
1: 660,000 .- \text { México, D. F. : La Direc- } \\
\text { ción, } 1958\end{array}$ \\
\hline & $\begin{array}{l}1 \text { mapa ; } 61 \times 113 \mathrm{~cm} \text {. } \\
\text { 1. Ferrocarril Chihuahua-Pacífico. } \\
\text { I. Título }\end{array}$ \\
\hline
\end{tabular}

G4421 Por terminar el primer número del bloque para mapas de los Es ta dos sep ten trio na les mexi ca nos en " 0 ", se es co ge de la pri me ra co lum na de la ta bla III el núme ro"1" para ma pas de ma te ria y se le suma a la úl tima cifra del primer número del bloque

.P3 La le tra y el nú me ro es co gi dos de la ta bla IV por ser un material cartográfico sobre ferrocarriles

1958 Fe cha de pu bli ca ción que coin ci de con la fe cha de la compilación de los datos

.M4 El nú me ro de Cut ter de asien to se gún la ta bla IV de autores (cuadro 3)

Para atlas y mapas de subáreas regionales, se escoge de la tabla III el tercer número, es decir 2 ó 7 . En este caso las subáreas se refieren a regiones y rasgos naturales, A-Z, cuando no están asig na dos nú me ros in di vi dua les. Tam bién como se verá ade lante, se asig nan los nú me ros 4 u 8 y 5 ó 9 de la ta bla III a su bá reas, siendo divisiones políticas mayores (condados, municipios, esta dos, pro vin cias, etc.) o ciu da des y pue blos de un área ma yor. Si la re gión o di vi sión po líti ca ma yor tie ne pro pio blo que de nú meros como ocurre en el caso de regiones y estados brasileños y mexicanos referente a los mapas (ejemplo 21) y entre varios otros paí ses, se les con si de ra como área ge ne ral. En tal caso, están asignados mayormente sólo tres o cuatro números a una región o estado porque las subdivisiones que corresponden al cuar to y/o al quin to nú me ro de la ta bla III tie nen sus pro pios bloques de nú me ros. Tam bién pu die ra ha ber atlas y ma pas so bre subá reas re gio na les por tema y en ton ces se agre ga ría se gún la ta bla IV una le tra y nú me ro más por el tema. Los ele men tos de la signatura topográfica consisten de:

\section{Para atlas}

- Clasificación por área

- Número de Cutter de la subárea + letra y número de materia en su caso + el Cutter de la autoridad

\section{Para mapas}

- Clasificación por área

- Número de Cutter de la subárea + letra y número de materia en su caso

- Fecha

responsable

- Fecha de publicación

Ejemplo 22

G1547

.P3C8E8

1958

\section{G1547}

Por ter mi nar el pri mer nú me ro del blo que para atlas de México en "5", se sustituye el número de la segunda columna de cifras de la tabla III, el "7" para atlas de regiones, rasgos naturales, etcétera, por el número correspondiente del bloque geográfico

.P3 El nú me ro de Cut ter para la Cuen ca del Pa pa loa pan según la tabla 4 de autores (cuadro 3)

C8 DelatablaIV desubdivisiones de mate ria, seescoge la letra y número de meteorología y climatología

E8 El número del asiento según la tabla 1 de autores

$1958 \quad$ La fecha de publicación del atlas

\section{Ejemplo 23}

G4512

. $\mathrm{Z3}$

1943

. $\mathrm{M} 4$
México. Dirección de Geografía, Meteorología e Historia. O ficina de Geografía. Plano de la serranía de Zacatecas: configuración estereofotogramétrica / levantado por la Dirección de Geografía, Meteorología e Historia, oficina de Geografía. - Escala 1:20,000. - México : La Dirección, 1943

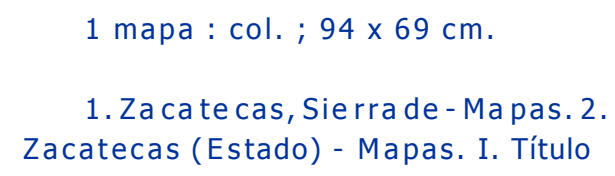

G4512 Por ter mi nar la pri me ra ci fra del blo que de nú me ros para ma pas del Es ta do de Za ca te cas en "0", se suma el nú me ro de la pri me ra colum na de ci fras de la ta bla III, el "2" para mapas de regiones, rasgos naturales, etcétera, al número base del bloque geográfico

El nú me ro de Cut ter de la re gión,Za ca tecas, (sie rra), según la tabla 4 de autores (cuadro 3) 
1943

Fecha

M4

El nú me ro de Cut ter del asien to prin ci pal se gún la tabla IV de autores

Ejemplo 24

G4682

.T4P6

1953

. $\mathrm{E} 8$

$$
\begin{aligned}
& \text { Estados Unidos. Aeronautical Chart Servi- } \\
& \text { ceTehuantepec Isth mus/Aeronautical } \\
& \text { Chart Service, U. S. Air Force. }-6 \text { th } \\
& \text { ed. - Escala } 1: 1,000,000 .- \text { Wa- } \\
& \text { shington, D. C. : The Service, } 1953 \\
& 1 \text { mapa : col. ; } 55 \times 73 \mathrm{~cm} \text {. - (World } \\
& \text { aeronautical chart ; } 644 \text { ) }
\end{aligned}
$$

1. Cartasaeronáuticas-Tehuantepec, Istmo de I. Título

G4682 Por terminar el número base del bloque para mapas de Estadosmeridionalesmexicanos en " 0 ", se suma el nú me ro de la pri me ra co lum na de ci fras de la ta bla III, el "2" para mapas de regiones, rasgos naturales, etcétera a la última cifra del número base

.T4 El nú me ro de Cut ter del Its mo de Tehuan te pec (cuadro 3)

P6

De la ta bla IV de sub di vi sio nes de ma te ria se es co ge la letra y número de cartas aeronáuticas

$1953 \quad$ Fecha

.E8 El nú me ro de Cut ter del asien to prin ci pal se gún la tabla 1 de autores

Se selecciona el cuarto número de la tabla III (3 u 8) para subáreas mayores que consisten de condados, municipios, estados, provincias, et cé te ra, al no te ner és tos un blo que pro pio de nú meros. Cuan do el mate rial car to gráfi co tra ta de sub di vi sio nes ad mi nistrativas aún más pequeñas dentro de la subárea, se insertan dos pun tos y el nú me ro "3" (:3) en tre los nú me ros de Cut ter de la su bárea ma yory la ad mi nis tra ti va. Se refie re el cla sifica dor alos ejem plos con cre tos de la Bi blio te ca del Con gre so (1976, p. 209) y de Immroth (1980, p. 174). Respecto al enfoque de materia para estas subáreas, se agrega en tal caso la letra y número correspondientes a la signatura topográfica.

Para atlas

- Clasificación por área

- Número de Cutter de la subárea + letra y número de materia en su caso + Cutter de la autoridad responsable

- Fecha de publicación

Ejemplo 25
G1548

.Y8E15G3

1980

Y8

E15

G3

1980

Ejemplo 26

G5283

.Z8

1964

.V4

G5283

- Clasificación por área

- Número de Cutter de la subárea + letra y número de materia en su caso

- Fecha

- Número de Cutter de la autoridad responsable
Garza Tarazona de González, Silvia Atlas arqueológico del Estado de Yucatán / Silvia Garza Tarazona de González y Edward Barna Kurjack Basco. - Escala $1: 200,000$. - Méxi co: SEP, Ins ti tu to Nacional de Antropología e Historia, Centro Regional del Sureste, 1980

1 atlas ( 2 v.) : il. (algu nos col.), mapas; $27-33 \times 45 \mathrm{~cm}$.

1. Yucatán - Arqueología - Mapas. 2. México - Arqueología - Mapas. II. Barna Kurjack Bas co, Ed ward. II. Instituto Nacional de Antropología e Historia. Centro Regional del Sureste. III. Título

Por terminar la última cifra del primer número del blo que para atlas de Mé xi co en "5", se sus ti tu ye de la segunda columna de cifras de la tabla III el número "8" para atlas de divisiones políticasmayores por la cifra correspondiente del bloque geográfico

El número de Cut ter de la di vi sión, en este caso Yucatán, según la tabla 4 de autores (cuadro 3 )

De la tabla IV de sub di vi siones de ma te ria, se es coge la letra y número de sitios arqueológicos

El nú me ro de Cut ter del asien to prin ci pal se gún la tabla 4 de autores

La fecha de publicación

Venezuela. Dirección de Cartografía $\mathrm{Na-}$ cional Carta geográfica del Estado de Zulia / Dirección de Cartografía Nacional. - Escala 1:500,000. - [Caracas] : La Dirección, 1964

1 mapa : col. ; $94 \times 77 \mathrm{~cm}$.

1. Zulia(Venezuela: Esta do)-Mapas. I. Título

Por terminar la última cifra del primer número del bloque para mapas de Venezuela en " 0 ", se escoge de la pri me ra co lum na de dí gi tos de la ta bla III el número"3" para ma pas de di vi siones políticas mayores y se suma al número base del bloque geográfico

Z8 El número de Cutter de la subdivisión mayor, Zulia (Venezuela : Estado) según la tabla 4 de autores (cuadro 3)

Fecha 
.V4 El número de Cutter del asiento según la tabla 4 de autores

Se aplica el quinto número del bloque que corresponde al 4 ó 9 de la ta bla III para su bá reas, sien do en este casociu da des o pueblos del área ma yor. Para la ma yo ría de los paí ses, los lu ga res están agru pa dos en el úl ti mo nú me ro de su blo que de nú me ros y no por la subdivisión política a que pertenecen.

Además la tabla III indica el uso de dos puntos para separar dos ti pos de su bá reas que se po drían lo ca li zar en una ciu dad. Es decir, la no ta ción : 2 se uti li za para ex pre sar una sub di vi sión re gional que no sea po lí ti ca, ya que para una con no ta ción de estaín dole se uti li za la no ta ción : 3 . La cla si fi ca ción para un mapa de es tas características tendría el siguiente orden: primero el número de clasificación propiamente dicho, en seguida el Cutter de la ciudad, después la notación :2 ó :3 y a continuación se indicaría el nú me ro de Cut ter pro pio de la su bá rea de que se tra te. La Bi bliote ca del Con gre so (1976, p. 210) e Immroth (1980, p. 174) ilustran con varios ejemplos este detalle. Se aplica la tabla IV también para ciudades y pueblos cuando el material cartográfico tiene un en fo que de ma te ria. En ca sos ge ne ra les, la sig na tu ra topográfica se construye así:

\section{Para atlas}

- Clasificación por área

- Número de Cutter de la subárea + letra y número de materia en su caso + Cutter de la autoridad responsable

- Fecha de publicación

Ejemplo 27

G1779

.S2S2

1956

$$
\begin{aligned}
& \text { Salvador (Brasil) D.T.C.M. Atlas parcial de } \\
& \text { Cidadede Sal va dor / Es ta do da Bahia, } \\
& \text { Prefeitura Municipal do Salvador, } \\
& \text { D.T.C.M. - Escala } 1 ; 1,333 .- \text { [Salva- } \\
& \text { dor] : D.T.C.M. , } 1956 \\
& 1 \text { atlas (26 p., } 41 \text { hojas de lams.) : } \\
& \text { mapas; } 24 \times 32 \mathrm{~cm} . \\
& \text { 1. Salvador (Brasil) - Mapas. I. Tí- } \\
& \text { tulo }
\end{aligned}
$$

G1779 Por terminar el último dígito del primer número del blo que para atlas de Bra sil en "5", se sus ti tu ye el número de la se gun da colum na de ci fras de la tabla III, el "9" para atlas de ciu da des y pue blos por el nú me ro correspondiente del bloque geográfico El nú me ro de Cut ter de la ciu dad se gún la ta bla 2 de autores (cuadro 3)

El número de Cutter del asiento principal según la misma tabla 2 de autores

$1956 \quad$ Fecha de publicación

\section{Ejemplo 28}

G4414

.S2P55

1942

.E8

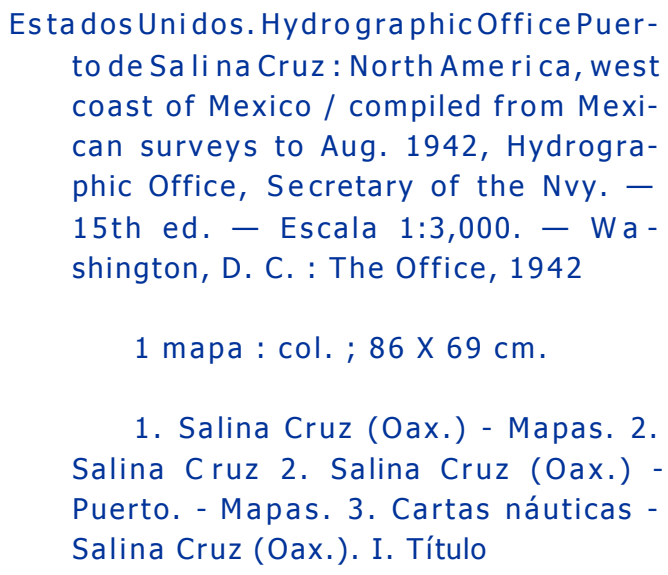
coast of Mexico / compiled from Mexican surveys to Aug. 1942, Hydrographic Office, Secretary of the Nvy. 15th ed. - Escala 1:3,000. - Washington, D. C. : The Office, 1942

1 mapa : col. ; $86 \times 69 \mathrm{~cm}$.

1. Salina Cruz (Oax.) - Mapas. 2. Salina Cruz 2. Salina Cruz (Oax.) Puerto. - Mapas. 3. Cartas náuticas Salina Cruz (Oax.). I. Título

G4414 Por terminar el último dígito del primer número del blo que para ma pas de Mé xi co en " 0 ", se suma el número de la primeracolumna de cifras de la tabla III, el "4", para ma pas de ciu da des y pue blos al nú me ro base del bloque geográfico

.S2 El número de Cutter de Sa li na Cruz se gún la ta bla 2 de autores

P55 DelatablaIV desubdivisiones de materia, se es coge la letra y número de puertos y servicios portuarios

$1942 \quad$ Fecha

.E8 El nú me ro de Cut ter del asien to prin ci pal se gún la tabla 2 de autores (cuadro 3 )

\section{Ejemplo 29}

G4414

.T6

1952

. $\mathrm{M} 4$

\author{
México. Depto. de Planeación y Fomento \\ de Carreteras Vecinales Plano de la \\ ciudad de Toluca / S.C.O.P., Departa- \\ mento de Planeación y Fomento de \\ Carreteras Vecinales. - Escala \\ 1:5,000. - México : El Depto., 1952 \\ 1 mapa; $77 \times 94 \mathrm{~cm}$. \\ 1. Toluca (Méx.) - Mapas. I. Título
}

G4414 Por terminar el primer nú me ro del blo que de nú meros para ma pas de Mé xi co en el dí gi to " 0 ", se suma la cifra de la primera columna de números de la Tabla III, el "4", para mapas de ciudades y pue blos, al número de base del bloque geográfico

El nú me ro de Cut ter de To lu ca se gún la ta bla 4 de autores (cuadro 3) 
El nú me ro de Cut ter del asien to prin ci pal se gún la tabla 4 de autores

Para colecciones demapas, se sus ti tu ye la fe cha con una "s" minúscula por la palabra "set" en inglés, siendo "serie" o "juego" en español seguida con la fracción representativa de la escala, pero sin las últimas tres cifras. Por ejemplo, con la escala 1:25,000 la designación en la signatura topográfica resulta en: s25. Por juegos de mapas con escala más grande de 1:1,000 se tra ta el de no mi na dor de la frac ción como si fue ra de ci mal y se le procede con un cero. Por ejem plo, con la es ca la 1:200 en la signatura topográfica sería: s02. En el caso de un juego de mapas con le tra y nú me ro de ma te ria o de un nú me ro de Cut ter de subárea, se coloca la designación de escala después de estos elementos, en donde iría la fecha sustituida:

\section{Ejemplo 30}

G5294

.B65

s5

I5

. B65

s5

\begin{abstract}
Institu to Geográfico Agustín Co dazziPla no de Bo go tá, D. E., Re pú bli ca de Colombia : plano aerofotogramétrico / elaborado para la Empresa de Acueducto y Alcantarillado de Bogotá, D. E. [por] el Instituto Geográfico Agustín Codazzi. - Escala 1:5,000. - [Bogotá] El Instituto, 1960
\end{abstract}

52 mapas; $82 \times 62 \mathrm{~cm}$.

1. Bogotá (Colombia) - Mapas. I. Empresa de Acueducto y Alcantarillado de Bogotá (D. E.). II. Título

G5294 Por terminar el primer número del bloque de cifras para ma pas de Co lom bia en el dí gi to "0", se suma el número de la primera columna de cifras de la tabla III, el "4" para ma pas de ciu da des y pue blos al nú mero de base del bloque geográfico

El nú me ro de Cut ter de Bo go tá, el pri me ro se gún la tabla de autores, el segundo ajustado de la tabla 5 para permi tirel asien to de otros lu ga res co lombia nos (cuadro 3)

El número de serie del mapa sin las últimas tres cifras de la escala

El nú me ro de Cut ter del asien to prin ci pal se gún la tabla 1 de autores

\section{CONCLUSIONES}

Existen otras clasificaciones de los mapas entre ellas uno usado precisamente en México, basado en la Proyección Universal de Mercator (U.T.M.) desarrollado por el Servicio de Mapas del Ejército de los Estados Unidos y adoptado internacionalmente por la Unión Geo dé si ca y Geo físi ca In ter na cio nal en Bru se las en 1951 (Karo 1955) utilizado por la INEGI (1983). Sin embargo, un sistema que ordena los mapas por números propios de serie como en este caso tiene que ser utilizado únicamente para sus mapas. Es otra ordenación que se aprovecha para clasificarlos pero su uti li dad no se ex tien de fá cil men te en in cluir otros ma pas de dis tin taín dole. Por lo in ter na cio nal que es la Cla si fi ca ción del Congreso, por ser un sistema siempre actualizado y por poder abarcar toda clase de mapas que el sistema del INEGI difícilmente pudiera hacer, se decidió recomendar esta clasificación para atlas y mapas. El procedimiento del INEGIesenteramente válido pero tendría que seguir siendo un caso especial adoptado para su pro pia co lec ción que no pue de in cluir otros asun tos fue ra de los suyos.

La Clasificación de Dewey (DD) que clasifica mapas en el número 912 también tiene sus dificultades por no poder tomar en cuen ta los ma pas por su tema sin ex ten der los nú me ros de ma siado. Por su pues to, para te ner co lec cio nes no muy gran des y comple jas pue de ser vir, como en el caso de las bi blio te cas pú bli cas. Una clasificación basada en Dewey, la Clasificación Decimal Universal (CDU) aunque incluye mapas, es un esquema demasiado intrincado. Tiene la desventaja del uso de muchos signos de puntuación, cada signo con diferente significado y resultan los núme ros declasificaciónim prác ti cos por serex cesivamente largos. Sin embargo, la utilizan extensamente en Europa (Larsgaard 1978).

Des pués de todo, to man do en cuen ta tiem po, cos tos y ser vi cio a usua rios, la Cla si fi ca ción del Con gre so ofre ce im por tan tes ventajas in clu si ve por su re la ti va sen cillez y sus nú me ros de cla si ficación que no resultan demasiado largos. A fin y al cabo, es el usuario quien cuenta. Adoptando una clasificación con serios defectos o con demasiadas complejidades que difícilmente los mismos clasificadores pudieran aplicar sin apropiar mucho tiempo en dominarla, crea graves problemas para después pensar en descartarla y utilizar otra. Aunque la Clasificación del Con gre so no es fá cil, no lle ga a las com pli ca cio nes de la CDU o las li mi ta cio nes de la del INE GI y al con tra rio de la DD, la Cla sificación del Congreso puede tomar en cuenta lo que es siempre importante, el enfoque temático de atlas y mapas. 


\section{BIBLIOGRAFIA CITADA}

ABELLB. Rober to “Ca ta loga ción y cla si fi ca ción de los ma pas en las bi blio te cas me xica nas", en Me mo rias del Se mina rio Nacional de Mapotecas. México Cetenal, 1977

BILINDEX; unalistabilingüe en es pañole in glés de en cabezamien tos de materia, Ro ber to Cabello Aran da na, exe cu tive di rector. Oakland, Calif., California Spanish Language Data Base, 1984

ESCAMILLA, Gloria Listade en cabezamientosdemateria. 2a ed. Méxi co, UNAM, Insti tu to de In vestigacio nes Biblio gráficas, 1978

IMMROTH, J. P. Immroth's guide to the Libraryof Congress clas sification. 3rd ed. by Lois Mai Chan. Li ttle ton, Colo., Li braries Unlimited, 1980

INS TITUTOCOLOMBIA NODEFOMENTODELAEDUCA CIONSUPERIOR Listade encabezamientosdemate riapara bibliotecas. Bogotá, Procultura, 1985

INS TITUTONACIONALDEESTADIS TICA, GEOGRAFIAEINFORMATICA ¿Cómo seorganizaunamapoteca? Mé xico, Secretaría de Programación y Presupuesto, Instituto Nacional de Estadística, Geografía e Informática, 1983

KARO, H. Arnold World mapping, 1954-1955. Washington, Industrial College of the Armed Forces, 1955

LARSGAARD, Mary Map librarianship; an introduction. Littleton, Colo., Libraries Unlimited, 1978

LIBRARY OF CONGRESS. Subject Cataloging Division LC classification, additions, and changes. 1928-, list 1-

LIBRARY OF CONGRESS. Subject Cataloging Division Classification, Class G, Geography, anthropology, folklore, manners and customs, recreation. 3rd ed. Washington, Library of Congress, 1954

LIBRARY OFCONGRESS. Subject Cata lo ging Di vi sion.Classification, Class G, Geo graphy, mapas, anthropologyandrecreation. 4th ed. Washington, Library of Congress, 1976

REGLASDECATALOGACIONANGLOAMERICANAS.2a ed. Wa shing ton, D.C., Or ga ni za ción de los Es ta dos Ame ri ca nos, 1983 